\title{
Experimental noninferiority trial of synthetic small-caliber biodegradable versus stable vascular grafts
}

\author{
Damiano Mugnai, MD, ${ }^{\mathrm{a}}$ Jean-Christophe Tille, MD, PhD, ${ }^{\mathrm{b}}$ Wojciech Mrówczyński, MD, ${ }^{\mathrm{a}, \mathrm{c}}$ \\ Sarra de Valence, MSc, ${ }^{\mathrm{d}}$ Xavier Montet, MD, ${ }^{\mathrm{e}}$ Michael Möller, $\mathrm{PhD},{ }^{\mathrm{d}}$ and Beat H. Walpoth, MD ${ }^{\mathrm{a}}$
}

Objective: Long-term evolution of polycaprolactone vascular prostheses has been investigated recently. The goal of this study was to evidence a noninferiority of such grafts compared with expanded polytetrafluoroethylene (ePTFE) implants in an aortic replacement model in the rat.

\begin{abstract}
Methods: Fourteen anesthetized Sprague-Dawley rats received an infrarenal aortic graft (biodegradable, $\mathrm{n}=8$; expanded polytetrafluoroethylene, $\mathrm{n}=6$ ) replacement (end to end; inner diameter, $2 \mathrm{~mm}$ ). Biodegradable grafts (polycaprolactone) were produced by random micro-/nanofiber electrospinning. After a median survival of 16.5 months, in vivo ultrasonography and angiography as well as postexplantation microcomputed tomography, histomorphometry, immunohistochemistry, and scanning electron microscopy were performed.
\end{abstract}

Results: Patency was $100 \%$ for polycaprolactone and $67 \%$ for ePTFE. No aneurysmal dilatation or stenoses were found in either group. Compliance was significantly higher for polycaprolactone compared with ePTFE $(8.2 \pm 1.0 \% / 100 \mathrm{~mm} \mathrm{Hg}$ vs $5.7 \pm 0.7 \% / 100 \mathrm{~mm} \mathrm{Hg} ; P<.01)$, but markedly reduced compared with adjacent native aortas and the control group. Histologically, low cellular in-growth was found in ePTFE whereas polycaprolactone showed significantly greater homogenous cellularity, producing an autologous extracellular matrix $(10.8 \% \pm 4.0 \%$ vs $32.1 \% \pm 9.2 \%, P<.0001)$. Morphometry showed $100 \%$ neo-endothelialization for both grafts with a totally confluent endothelial coverage for polycaprolactone grafts by scanning electron microscope. More intimal hyperplasia was found in ePTFE compared with polycaprolactone grafts. Calcification was higher in ePTFE than in polycaprolactone grafts $(15.8 \%$ vs $7.0 \%, P=.04)$ and was absent in controls.

Conclusions: Outcomes of synthetic biodegradable nanofiber polycaprolactone grafts are not inferior compared with the clinically used expanded polytetrafluoroethylene grafts after long-term implantation in the rat aorta. Moreover, these implants show better patency, compliance, endothelialization, and cell in-growth, and less intimal hyperplasia and calcification than their counterparts. (J Thorac Cardiovasc Surg 2013;146:400-7)

Supplemental material is available online.

The preferred choice for small-diameter vessel replacements is autologous vascular material because it has the

From the Service of Cardiovascular Surgery ${ }^{\mathrm{a}}$ and Service of Clinical Pathology, ${ }^{\mathrm{b}}$ University Hospital, Faculty of Medicine, Geneva, Switzerland; the Department of Pediatric Cardiac Surgery, ${ }^{c}$ Poznan University of Medical Sciences, Poznan, Poland; the School of Pharmaceutical Sciences, ${ }^{\mathrm{d}}$ University of Geneva, University of Lausanne, Lausanne, Switzerland; and the Laboratory of Molecular Imaging, ${ }^{e}$ Department of Radiology, Faculty of Medicine, University of Geneva, Geneva, Switzerland.

This study was supported by the Swiss National Science Foundation (FNS-Subside FN 320000-119822/1) and institutional funds of the Cardiovascular Research Group.

Disclosures: Authors have nothing to disclose with regard to commercial support.

D.M., J.-C.T., and W.M. contributed equally to this study.

Received for publication May 18, 2012; revisions received July 31, 2012; accepted for publication Sept 21, 2012; available ahead of print Oct 24, 2012.

Address for reprints: Beat H. Walpoth, MD, Service of Cardiovascular Surgery, University Hospital of Geneva, 4, rue Gabrielle-Perret-Gentil, 1211 Geneva 14, Switzerland (E-mail: beat.walpoth@hcuge.ch).

$0022-5223 / \$ 36.00$

Copyright (c) 2013 by The American Association for Thoracic Surgery

http://dx.doi.org/10.1016/j.jtcvs.2012.09.054 best clinical outcome compared with currently available vascular prostheses. ${ }^{1}$ Biologically stable synthetic grafts such as expanded polytetrafluoroethylene (ePTFE) and polyethylene terephthalate (Dacron) grafts are used successfully for the replacement of large-diameter vessels, but are prone to thrombosis and intimal hyperplasia development when used as small-diameter vessel replacements $(<6 \mathrm{~mm}){ }^{2}$ Because autologous vascular material is not always available or appropriate, it would be greatly beneficial to have a shelf-ready alternative. A significant research effort has been led in this direction for the past 20 years, but no real solutions have emerged. ${ }^{3}$

Our in vivo tissue engineering approach to resolve the problem of small-diameter vascular grafts is based on the use of a synthetic, biodegradable, electrospun, nanoscaffold to serve as a guide for the regeneration of a new vessel with autologous cells and extracellular matrix (ECM). The use of a biodegradable material avoids problems linked to longterm residency of synthetic materials in arterial circulation, such as foreign body reaction and infections. ${ }^{4}$ Other approaches to small-diameter vascular prostheses have also been explored, ${ }^{5}$ such as biodegradable scaffolds with preimplantation cell seeding with ${ }^{6,7}$ or without ${ }^{8}$ bioreactor maturation, or cell sheet-based tissue engineered grafts. ${ }^{9}$ 


\section{Abbreviations and Acronyms \\ $\mathrm{ECM}=$ extracellular matrix \\ ePTFE $=$ expanded polytetrafluoroethylene \\ $\mathrm{PCL}=$ polycaprolactone}

Nevertheless, being shelf ready is important for clinical use, which is not the case for the these cell-based grafts.

In this study, the long-term results of a biodegradable synthetic vascular graft were evaluated and compared with the commercially available ePTFE graft as an aortic replacement in the rat model. The evaluated graft was a micro- and nanofiber-based 2-mm-inner diameter vascular prosthesis made from randomly oriented electrospun polycaprolactone (PCL) fibers. Its highly porous structure makes it suitable for cell infiltration and allows uniform degradation throughout the graft while natural tissues regenerate. In previous studies carried out by our group, this graft was characterized and evaluated in detail, both in vitro and in vivo, in the rat abdominal aorta replacement model for up to 6 months. ${ }^{10,11}$ In a recent publication by our group, we demonstrated the long-term evolution of PCL grafts (18 months). ${ }^{12}$ They showed very promising results, with rapid endothelialization, limited neointima formation, and graft patency throughout the entire follow-up period. However, despite this good performance, PCL has some well-established limitations in the long term (eg, calcification, changes in cellular density). ${ }^{12}$ Little is known whether these drawbacks are comparable with problems encountered during long-term implantation of ePTFE grafts, which are commonly used clinically.

The objective of our study was to prove the noninferiority of biodegradable PCL grafts compared with stable ePTFE implants of the same caliber in a rat model during a period up to 18 months. To achieve our goal, both grafts were compared with regard to patency, aneurysmal dilation, cell invasion, endothelialization, intimal hyperplasia, compliance, and calcification.

\section{METHODS}

The experimental protocol was approved by the Animal Experiments Ethical Committee of the University of Geneva (Protocol:06/52) and the Veterinary Office of the State of Geneva, Switzerland (1081/3232/II), and carried out in conformity with the Guide for Care and Use of Laboratory Animals. ${ }^{13}$

\section{Preparation of Vascular Grafts}

The 2-mm-inner diameter biodegradable vascular grafts made from PCL were obtained by electrospinning a solution of $15 \%$ w:v PCL $\left(\mathrm{M}_{\mathrm{w}}=80,000 \mathrm{~g} / \mathrm{mol}\right.$; Sigma, Hamburg, Germany) and $\mathrm{CHCl}_{3} / \mathrm{EtOH}$ (7:3, v:v) (Reidel-de-Haën, Seelze, Germany; Hänseler, Herisau, Switzerland) as described previously in detail. ${ }^{10,11}$ In brief, the solution is ejected from a charged needle $(20 \mathrm{kV})$ at a flow rate of $12 \mathrm{~mL} /$ hour. The presence of the high electrical field creates micro- and nanopolymer fibers, which are collected onto a 2-mm-diameter grounded mandrel $20 \mathrm{~cm}$ from the ejecting needle, which is both rotating and translating to ensure a homogenous random deposition of the fibers. All grafts had a mean fiber diameter of $2.2 \pm$ $0.6 \mu \mathrm{m}$, a maximum tensile stress of $4.1 \pm 0.5 \mathrm{MPa}$, and a maximum tensile strain of $1092 \pm 28 \%$ after sterilization by $\gamma$-radiation ( $25 \mathrm{kGy})$. Commercially available ePTFE grafts served as controls (30- $\mu \mathrm{m}$ thru-pore, 2-mm inner diameter; Atrium, Mijdrecht, The Netherlands).

\section{Implantation}

Eight PCL grafts and 6 ePTFE grafts were implanted in 14 male Sprague-Dawley rats $(275 \mathrm{~g})$ under $2 \%$ isoflurane mask anesthesia after induction by $5 \%$ isoflurane. The rat abdomen was opened with a midline laparotomy incision, and the infrarenal abdominal aorta was isolated by blunt and sharp dissection. After proximal and distal clamping below the renal arteries and above the aortoiliac bifurcation, a 1-cm segment of the aorta was resected in a beveled way. Polycaprolactone or ePTFE grafts (20 mm long) were implanted with 10-0 nylon interrupted sutures using an operative microscope at 10 times magnification under sterile conditions. Surgical handling and suture retention were better than ePTFE grafts, and hemostasis of PCL grafts was excellent after declamping the aorta.

\section{Patency, Stenosis, and Aneurysmal Dilatation}

At the end of the follow-up period, the following studies were performed after the animals were sacrificed: in vivo noninvasive compliance measurements of the graft and the aorta as well as digital subtraction angiography (General Electric Cardiac Series, 9800; Salt Lake City, Utah). The animals were sacrificed with a pentobarbital overdose, and the infrarenal proximal and distal segment of the abdominal aorta, including the graft, was explanted. Six sex- and age-matched rats, which served as controls, underwent the same examinations.

\section{In Vivo Compliance}

Compliance is a measure of the elastic deformation of a material in response to pressure. According to the American National Standards Institute guidelines, ${ }^{14}$ it is calculated as follows:

$$
\% \text { Compliance } / 100 \mathrm{~mm} \mathrm{Hg}=\frac{\left(R_{p_{2}}-R_{p_{1}}\right) / R_{p_{1}}}{p_{2}-p_{1}} \times 10^{4},
$$

where $p_{1}$ is the lower pressure value, $p_{2}$ is the higher pressure value (measured in millimeters of mercury), and $R_{p_{1}}$ and $R_{p_{2}}$ are the vessel inner diameters at the respective pressures. Compliance of the implanted grafts and the adjacent native aorta were measured in vivo in all rats before graft explantation. Rats were kept under narcosis with a $2 \%$ isoflurane mask while high-resolution ultrasound imaging (Vevo 770; Visualsonics, Toronto, Ontario, Canada) was performed. One hundred frame videos at a $71-\mathrm{Hz}$ frame rate were recorded at standard locations (mid graft, and proximal and distal native aorta). The rats' blood pressure was measured simultaneously with a noninvasive tail cuff system (CODA; Kent Scientific Corporation, Torrington, Conn). Using standard image processing software (ImageJ, ver. 1.44k, 2010; National Institutes of Health, Bethesda, Md), vessel diameters were measured on each frame. Average systolic and diastolic diameters were calculated from approximately 10 heart cycles. These data were then used to calculate the compliance according to the previous formula.

\section{Histology and Morphologic Quantitative Analysis}

For the histologic evaluation, explanted grafts with both anastomoses were fixed in $4 \%$ formaldehyde for 24 hours and embedded in paraffin. Longitudinal, $4-\mu \mathrm{m}$ thick histologic sections were stained with hematoxylin-eosin, Miller-Masson for elastin and collagen staining, and von Kossa for calcium deposition. Immunohistochemistry was performed with antiCD34 antibody (sc-7045, dilution, 1:200; Santa Cruz Biotechnology, Inc, Heidelberg, Germany) for endothelium and neo-angiogenesis staining, 
and antismooth muscle actin antibody (N1584, dilution, 1:800; Dako Schweiz Ag, Baar, Switzerland) for smooth muscle cells. Histologic slides were scanned numerically in their totality at 10 times magnification (Mirax scanner; Carl Zeiss MicroImaging $\mathrm{GmbH}$, Göttingen, Germany) for quantifications with ImageJ (ImageJ, ver. 1.44k, 2010). The following measurements were acquired from each slide: percent endothelial coverage (percentage of the graft luminal surface covered by endothelial cells), neointima coverage (percentage of the graft length covered with neointima), average neointima thickness (the area between the endothelial layer and the graft surface, normalized by the neointima length and measured in micrometers), percent transmural cellular in-growth (percentage of the graft area densely populated by host cells from the adventitial tissue toward the luminal side).

In addition, scanning electron microscopy (Philips XL20 SEM, Eindhoven, The Netherlands) was used to scan longitudinal sections of explanted grafts. Samples were prepared with standard dehydration and gold coating techniques.

\section{Evaluation of Calcification}

Microcomputed tomographic ex vivo scans of the grafts and control aortas were acquired at an $18-\mu \mathrm{m}$ resolution on a Skyscan-1076 micro-CT (Skyscan, Aartselaar, Belgium). Cross-sectional images were reconstructed using a classic Feldkamp cone-beam algorithm. ${ }^{15}$ Calcium volumes were extracted automatically based on densities superior to 200 Hounsfield units. Calcium percentages were calculated as the volume of calcium divided by the total volume of the prosthesis/aorta.

\section{Statistical Analysis}

Results are expressed as a mean \pm standard deviation. Intergroup comparisons were performed with the use of Mann-Whitney $U$ test. The Fisher exact test was used for binary variables. Totally occluded grafts were excluded from the comparisons. A 2 -tailed $P$ value $<.05$ was considered significant in all tests. $P$ values between .05 and .1 marked a trend toward statistical significance.

\section{RESULTS}

\section{Follow-up}

The mean follow-up time was $14.8 \pm 2.9$ months and $16.0 \pm 3.1$ months in the PCL and ePTFE groups, respectively $(P=.17$; Table 1$)$. One rat implanted with a PCL graft developed severe breast tumors as a result of aging and was sacrificed, although the graft was patent.

\section{Patency, Stenosis, and Aneurysmal Dilation}

The PCL grafts had excellent patency rates (100\%), but 2 of the ePTFE grafts were occluded at the conclusion of the study, yielding a patency of $67 \%(P=.46$; Table 1 and Figure 1, A). By angiographic assessment, no signs of stenosis could be detected for the PCL grafts, and nonrelevant $(<50 \%$ obstruction) stenoses were found for all patent ePTFE grafts (Figure 1, $B$ and $C$ ). Neither the PCL grafts nor the ePTFE grafts showed signs of aneurysmal dilation. Angiographies of the control aortas revealed $100 \%$ patency, no stenosis, and no aneurysmal dilation (Figure 1,D).

\section{In Vivo Compliance}

The diastolic and systolic inner diameters were calculated for the middle of the grafts and the adjacent proximal
TABLE 1. Comparison between PCL and ePTFE grafts

\begin{tabular}{|c|c|c|c|}
\hline Parameter & PCL $(n=8)$ & ePTFE $(n=6)$ & $P$ value \\
\hline Survival, months $\pm \mathrm{SD}$ & $14.8 \pm 2.9$ & $16.0 \pm 3.1$ & .17 \\
\hline Patency, \% & 100 & 67 & .46 \\
\hline $\begin{array}{l}\text { Compliance, } \% / 100 \mathrm{~mm} \mathrm{Hg} \\
\quad \pm \mathrm{SD}\end{array}$ & $8.2 \pm 1.0$ & $5.7 \pm 0.7$ & .01 \\
\hline Endothelialization, $\% \pm \mathrm{SD}$ & $100 \pm 0.0$ & $99.6 \pm 1.0$ & .92 \\
\hline Neointima coverage, $\% \pm \mathrm{SD}$ & $67.9 \pm 17.1$ & $77 \pm 15.6$ & .25 \\
\hline $\begin{array}{l}\text { Average thickness of neointima, } \\
\mu \mathrm{m} ; \pm \mathrm{SD}\end{array}$ & $51.6 \pm 20.7$ & $76.9 \pm 36.9$ & .09 \\
\hline Cellular in-growth, $\% \pm \mathrm{SD}$ & $32.1 \pm 9.2$ & $10.8 \pm 4.0$ & $<.001$ \\
\hline Calcification, $\% \pm \mathrm{SD}$ & $7.0 \pm 5.0$ & $15.8 \pm 3.2$ & .04 \\
\hline
\end{tabular}

$\overline{P C L}$, Polycaprolactone; ePTFE, expanded polytetrafluoroethylene; $S D$, standard deviation.

and distal native aorta of both grafts (Figure E1). The compliance of PCL implants was significantly greater $(P=.011)$ than $\mathrm{PTFE}$ grafts: $8.2 \pm 1.0 \% / 100 \mathrm{~mm} \mathrm{Hg}$ versus $5.7 \pm 0.7 \% / 100 \mathrm{~mm} \mathrm{Hg}$, respectively. However, the compliance of both PCL and ePTFE grafts was significantly less than their adjacent native aortas and control nonoperated aortas (Figure E2 and Table 1).

\section{Morphologic Analysis of PCL Grafts}

Polycaprolactone grafts were lined completely with endothelium, which was confirmed by CD34 immunohistochemistry (Figure 2, $A$ and $D$ ), and populations of homogenously distributed cells were present throughout the graft material (Figure 2, B). In the graft bodies, numerous macrophages, with a few myofibroblasts near the luminal side and neoangiogenesis could be found. The grafts were bordered by a mild foreign body reaction, but there were no signs of chronic inflammation. On the luminal side, spindle-shaped cells beneath the endothelium formed a neointimal layer (Figure 2, C). Focal calcium depositions were found at the interface of the prosthesis and intimal hyperplasia, mainly in proximity to the anastomoses, but seldom invading the graft body (Figure 2,C). Miller-Masson staining shows that new ECM was produced by the cells in and on the luminal surface of the graft. Collagen deposition was found both in the body of the graft (Figure 2, $E$ ), and elastin fibers were found in the neointima (Figure 2,E). Smooth muscle cells and myofibroblasts were labeled with anti- $\alpha$ smooth muscle actin antibody and were found in the neointima layers, but were scarce in the graft body (Figure 2, F).

\section{Morphologic Analysis of the Expanded Polytetrafluoroethylene Grafts}

Histologic evaluation of the ePTFE grafts showed that cell invasion was very limited and was comprised of only a few lymphocytes, fibroblasts, and rare capillaries (Figure 3,A). At the adventitial interface, no foreign body reaction was found. Endothelial cells covered most of the luminal surface, and neointimal layers under the 


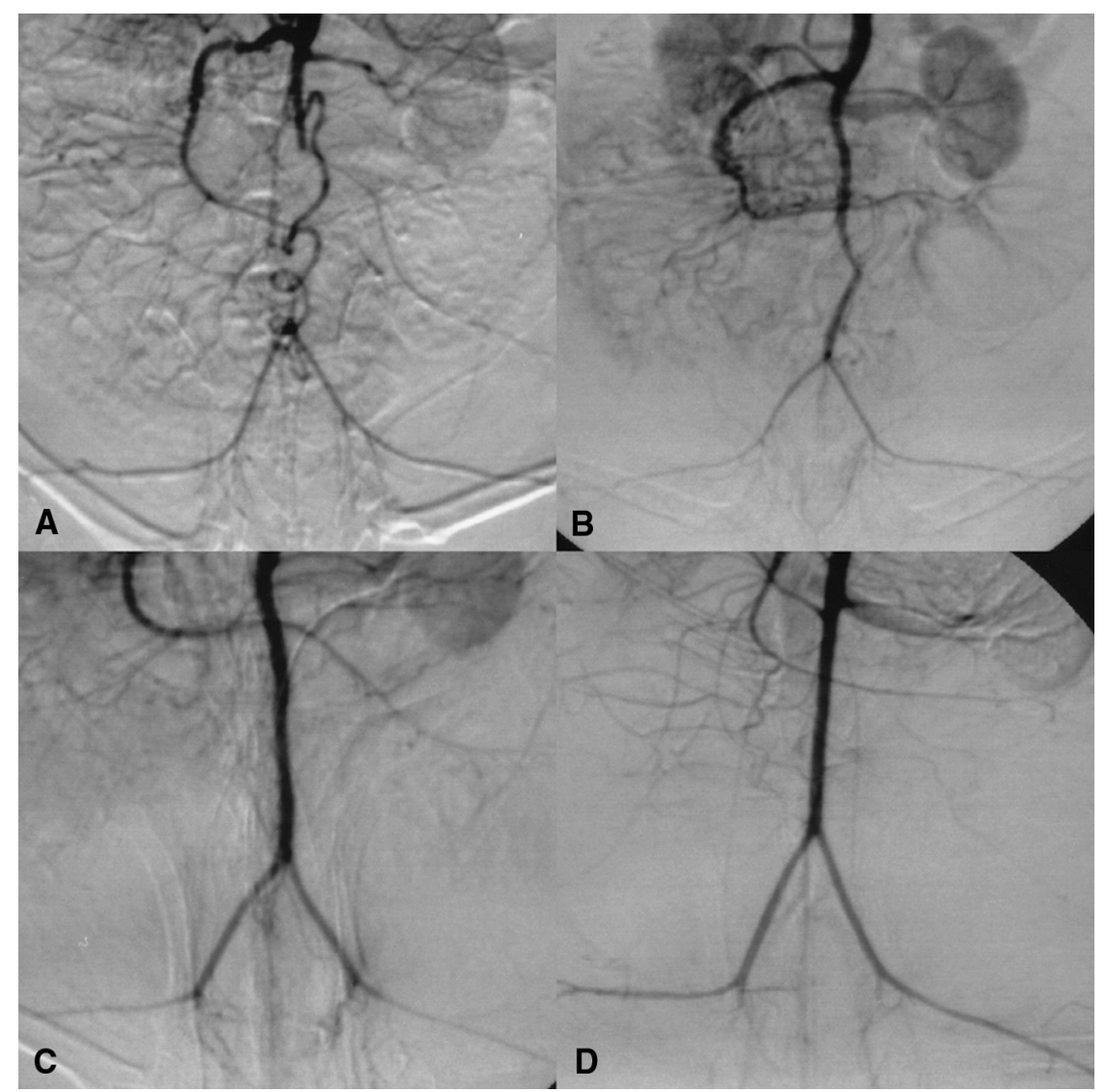

FIGURE 1. Evaluation of patency after 1-year implantation. (A) In vivo angiography before explantation reveals 1 obstructed ePTFE graft where irrigation of the lower limbs is ensured by a collateral vessel. (B) In patent ePTFE grafts, flow is reduced, with signs of insignificant stenosis $(<50 \%)$. (C) Patency of PCL grafts was very good with no stenosis or aneurysmal dilation. (D) The patent native aorta of a control rat.

endothelium were present (Figure 3,B). Extracellular matrix deposition was limited to collagen on the inner side of the graft in addition to collagen and elastin in the neointima layers (Figure 3,C). Several areas of calcium deposition were found only in the graft body and were never seen in the neointima (Figure 3, D).

Scanning electron microscopic images show confluent endothelialization of PCL grafts (Figure 2, A), but areas of incomplete endothelial coverage were found on ePTFE samples (Figure 3, E; insert). In addition, some areas of microthrombi were present on the luminal surface of 1 ePTFE graft, and not on PCL grafts.

\section{Histologic Morphometric Analysis}

Longitudinal histologic cross-sections revealed $100 \%$ endothelialization in PCL and $99.6 \% \pm 1.0 \%$ endothelialization in ePTFE grafts (Table 1). However, the minimal value of endothelium coverage for ePTFE implants was $97 \%$, confirming incompleteness of coverage in some grafts. The neointimal coverage did not differ significantly in the studied groups, and was $67.9 \% \pm 17.1 \%$ and $77.0 \% \pm 15.6 \%$ in PCL and ePTFE implants, respectively $(P=.25)$. However, the average thickness of neointima was less in the PCL group $(51.6 \pm 20.7 \mu \mathrm{m})$ than in the ePTFE group $(76.9 \pm 36.9 \mu \mathrm{m}$; Figure E3, $A$ ). This result shows a trend toward statistical significance $(P=.09)$. Transmural cellular in-growth was significantly greater $(P<.001)$ in PCL prostheses $(32.1 \% \pm$ $9.2 \%)$ than in the ePTFE counterparts $(10.8 \% \pm 4.0 \%$; Table 1 and Figure E3, $B$ ).

\section{Calcium Deposition}

Calcifications of the PCL grafts were located at the interface of the neointima-graft lumen (Figure 2, C) whereas in ePTFE prostheses they were observed only in the body of the implant (Figure 3,D). Calcium depositions were confirmed by microcomputed tomographic imaging of both grafts (Figure 4), which revealed several areas of calcification in all PCL and all ePTFE grafts, with the major ones often positioned near an anastomosis. Quantitative volumetric assessment showed that calcification in ePTFE grafts was significantly greater $(P=.04)$ than in PCL grafts, with an average calcium content of $15.8 \% \pm 3.2 \%$ compared with $7.0 \% \pm 5.0 \%$ for the PCL-based grafts (Table 1 ). Native aortas from the control animals had no detectable calcifications. 


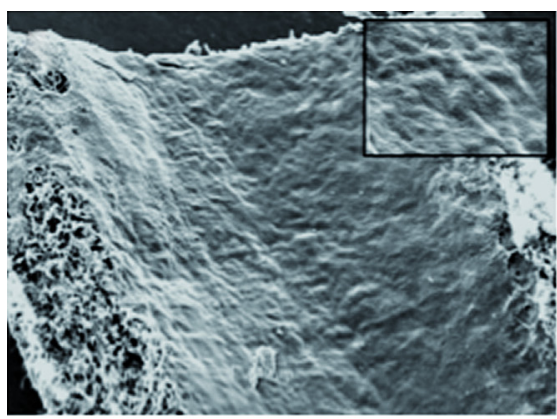

A

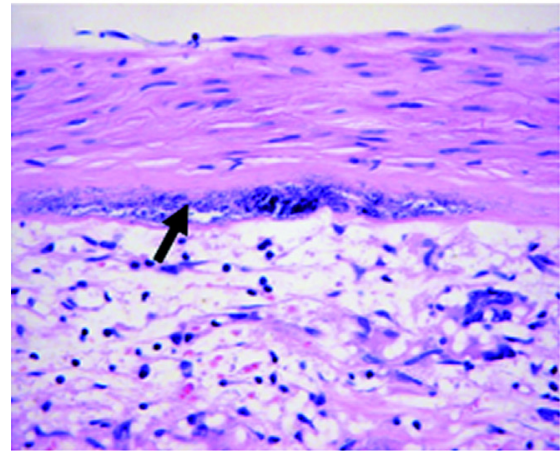

C

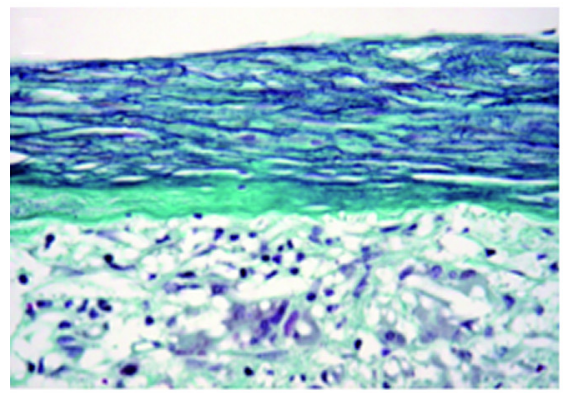

E

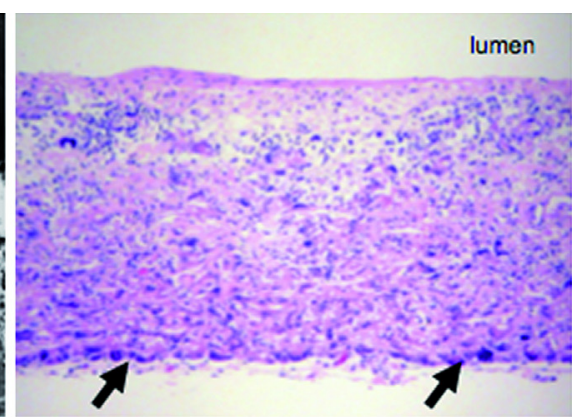

B

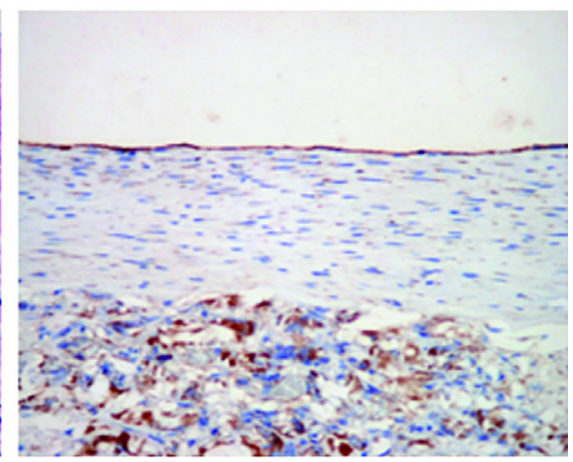

D

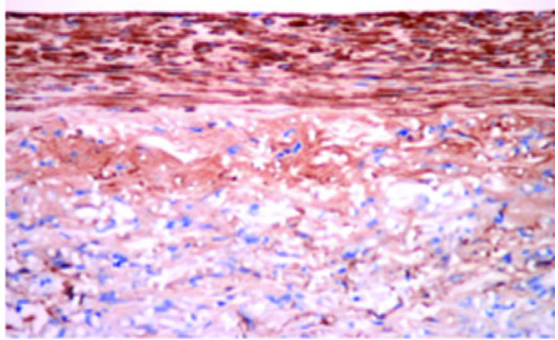

$F$

FIGURE 2. Morphologic analysis of PLC grafts. (A) Scanning electron micrographic image of the lumen of the PCL graft after explantation showing complete endothelialization. (B) Longitudinal section of the graft wall showing homogenous cellular infiltration giant cells on the periphery (arrows; hematoxylin-eosin staining, $100 \times$ magnification). (C) Neointima with spindle-shaped cells above a calcified area (arrow). An endothelium is present on the luminal side (hematoxylin-eosin staining, $200 \times$ magnification). (D) Immunohistochemistry anti-CD34 labeling endothelial cells on the luminal side (200× magnification). (E) Elastin deposition in the neointimal layers is revealed in blue and collagen deposition is revealed in green by MillerMasson staining (200× magnification). (F) Immunohistochemistry anti- $\alpha$ smooth muscle actin demonstrating positivity in spindle-shaped cells forming the neo-intima (200× magnification).

\section{DISCUSSION}

In the quest for a small-diameter vascular prosthesis, biodegradable synthetic grafts have become an important area of research. In our study, we present results of 2-mm-inner diameter PCL grafts implanted in the rat aorta for 12 to 18 months (thus, the average trial period of 14.8 months represents approximately 18 years in a human), and compare them with ePTFE grafts and control native aortas. The performance of PCL is equal to ePTFE in our study and even better with regard to patency, compliance, intimal hyperplasia, cellular in-growth, and calcification (Table 1).
After the follow-up period, all 8 PCL grafts evaluated were patent and showed no signs of stenosis, whereas 2 ePTFE grafts out of 6 were thrombosed. Microthrombi could also be spotted on the luminal surface of 1 patent ePTFE graft by scanning electron microscopy. In a previous report, we showed that endothelialization of our PCL grafts occurs much more rapidly than on ePTFE grafts, and remains stable and confluent for all further time points. ${ }^{11,12}$ Maintaining a stable antithrombotic surface is essential for long-term success of a vascular prosthesis, and the best surface is a functional endothelium. ${ }^{16}$ As seen in our 

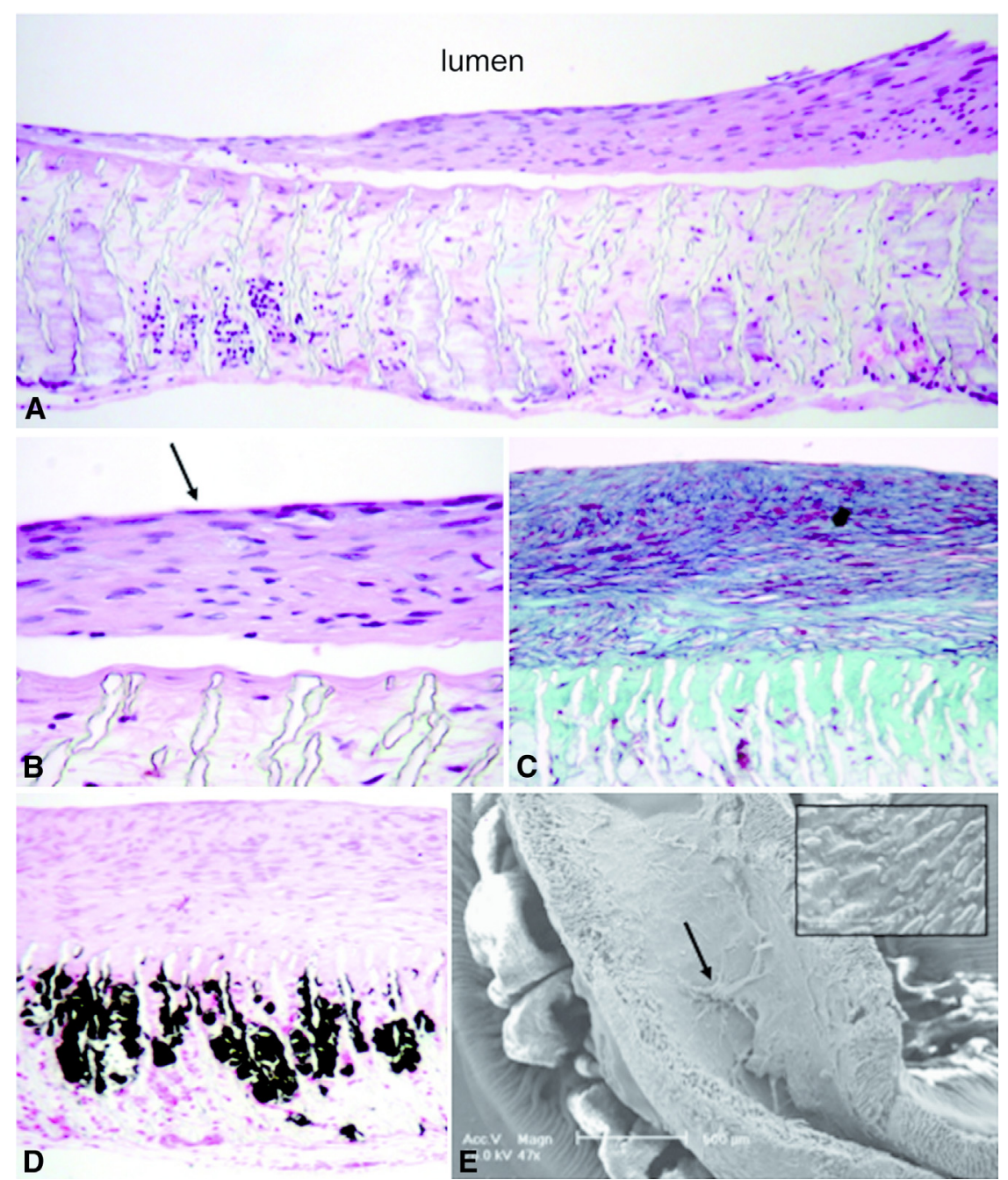

FIGURE 3. Morphologic analysis of expanded polytetrafluoroethylene grafts. (A) Longitudinal section of the graft wall with neointima formation, limited cellular infiltration in the graft body, and no giant cell reaction (hematoxylin-eosin staining, $100 \times$ magnification). (B) Neointima formation under the endothelium (arrow) with no signs of calcification (hematoxylin-eosin staining, 200× magnification). (C) Miller-Masson staining revealing elastin fibers in blue in the neointima and collagen depositions in green in the inner part of the graft (200× magnification). (D) Von Kossa staining showing, in black, the calcifications in the graft body (100× magnification). (E) Scanning electron microscopic image of the lumen of the graft showing incomplete endothelialization (insert) and microthrombi.

results, endothelial coverage of the ePTFE graft is not completely confluent; at 12 to 18 months, some areas are lacking an endothelium. This makes the ePTFE surface prone to thrombosis and may explain the lesser patency rate and microthrombi observed in this study. Therefore, electrospun PCL grafts show a better tendency toward long-term thrombus resistance.

As a biodegradable graft evolves over time toward a fully regenerated vessel, the biomaterial degrades and the mechanical strength of the synthetic structure decreases. A decrease in molecular weight of approximately $80 \%$ was found at 18 months after implantation. ${ }^{12}$ The ideal biomaterial should degrade in conjunction with natural ECM deposition for the conservation of structural mechanical properties and the prevention of aneurysmal dilation. After
$>1$ year, despite signs of degradation with macrophage infiltrate seen histologically, our biodegradable PCL grafts showed no signs of aneurysmal dilations or mechanical weaknesses. This is probably a result, in part, of the slow degradation rate of PCL and of the collagen accumulation in the graft body. Although it is difficult to know to what proportion the ECM is contributing to the net mechanical strength, we know that up to 18 months, our degrading graft has sufficient strength to withstand arterial pressure.

After 1.5 years of implantation, local thickening of the intima under the endothelium could be found in all PCL and ePTFE grafts. Because the average thickness of this layer in PCL is low and no flow obstruction can be observed, the neointima present does not seem to be evolving toward a pathologic condition. This is not necessarily true in the 


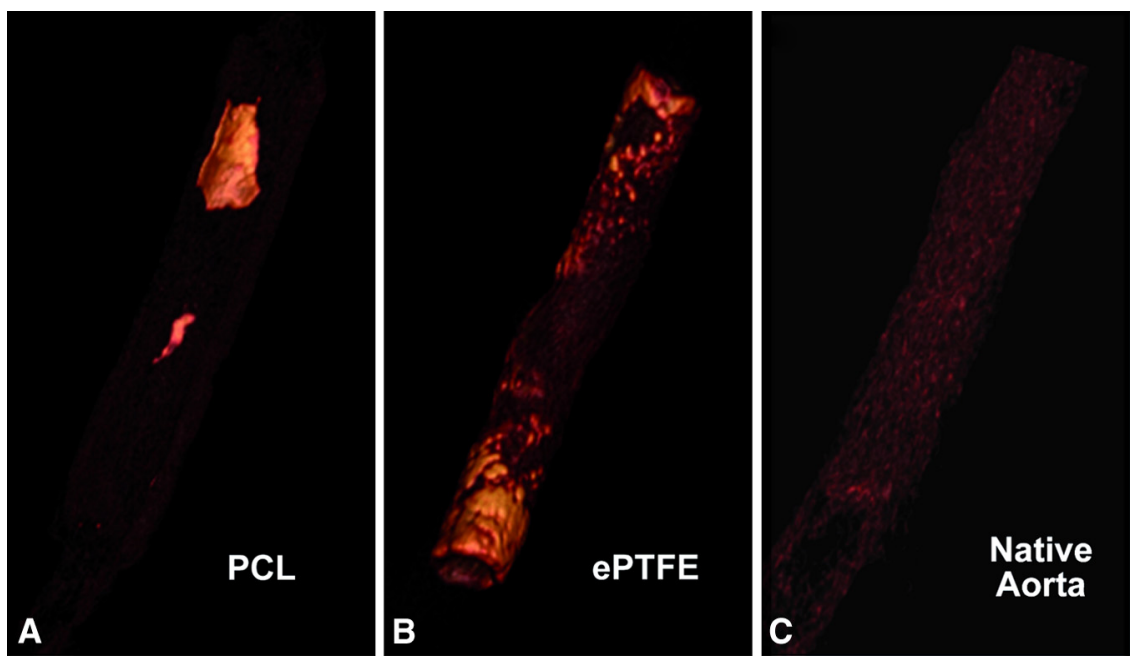

FIGURE 4. Evaluation of calcium content. The microcomputed tomographic 3-dimensional images of the full grafts taken ex vivo immediately after explantation reveal calcifications in yellow and noncalcified tissues in dark brown. Some localized calcifications are found in PCL grafts (A) whereas ePTFE grafts are subject to more extended calcifications (B), and no calcified tissues are detected in the control native aorta (C). PCL, Polycaprolactone; ePTFE, expanded polytetrafluoroethylene.

case of ePTFE implants, which presented increased thickness of neointima, potentially predisposing to further occlusion, as found in this group of implants.

In terms of tissue regeneration, after 12 to 18 months, the PCL graft bodies are populated mainly by macrophages, adventitial giant cells, and fibroblasts, some of which are myofibroblasts. Moreover, limited neo-angiogenesis is demonstrable and is a favorable phenomenon compared with ePTFE implants. With regard to the latter, neoangiogenesis is not as important because it is a stable graft, but it becomes crucial for all biodegradable implants in which tissue regeneration provides appropriate mechanical resistance and what is sought-integration with the host.

Biomaterial-related calcification of cardiovascular implants has been described previously ${ }^{17,18}$ and is not only a rat-specific reaction, because it is detected in most clinically implanted ePTFE grafts, even after short periods of implantation, if it is sought. ${ }^{19}$ Biomaterial-related calcification is also observed in our study in both the PCL and ePTFE grafts. The microcomputed tomographic scans show that total calcium content is significantly more important in the ePTFE grafts than in the PCL grafts.

As demonstrated in this article, we can determine the compliance in vivo by a very effective and noninvasive method, opening up the possibility of following the compliance of implanted grafts. In vitro compliance is inherent to the material and the manufacturing process, and will change after implantation as a result of degradation, tissue ingrowth, ECM formation, and calcification. Our results show a difference in elasticity, indicating a compliance mismatch for PCL grafts compared with the adjacent native aorta. The main problem associated with compliance mismatch is the development of intimal hyperplasia, which can lead to graft failure. ${ }^{20}$ The better patency of PCL implants compared to ePTFE grafts could be explained in part by improved vascular compliance that potentially diminished unfavorable stimulus for intimal hyperplasia formation

\section{Study Limitations}

This study is based on a limited number of animals because of ethical veterinary considerations (3R Rule) and governmental regulations on the number of experimental animals used. We kept our study follow-up survival up to 18 months, which represents almost a lifespan for a rat. Further observation becomes very hazardous because of possible late appearance of malignancies and other diseases in old rats. Abdominal aorta replacement in the rat is an established model with a limitation of representing a high-flow/ high-shear rate situation despite the small size of our implants. Obviously, different mechanical and rheologic conditions are found in peripheral arterial vessels. Therefore, a large-animal model such as porcine carotid artery replacement should be used as a preclinical pivotal study. However, our model seems to be a simple and robust method for graft testing in small animals. Furthermore, enhancement of graft compliance is an important issue to reduce compliance mismatch between our prostheses and a native artery.

\section{CONCLUSIONS}

Outcomes of synthetic, biodegradable, nanofiber, electrospun PCL grafts are not inferior compared with the clinically used stable ePTFE grafts after long-term implantation in the rat aorta. Of the 8 PCL grafts evaluated, all were patent with no signs of stenosis; had no aneurysmal dilation despite 
degradation; were fully reendothelialized and thus resistant to thrombosis; had limited, nonprogressing intimal hyperplasia; and had much less calcification as well as better compliance than ePTFE grafts. These grafts may, therefore, have the potential to provide a better clinical outcome for smallvessel revascularization procedures than ePTFE grafts.

We thank Unn Lutzen and Patricia Gindre for histologic and immunohistochemistry preparations, Marie-Claude Reymond for scanning electron microscopy preparations, and Jean-Pierre Giliberto for assistance during surgery.

\section{References}

1. Tomizawa Y. Vascular prostheses for aortocoronary bypass-grafting: a review. Artif Organs. 1995;19:39-45.

2. Walpoth BH, Bowlin GL. The daunting quest for a small diameter vascular graft. Exp Rev Med Device. 2005;2:647-51.

3. Zilla P, Bezuidenhout D, Human P. Prosthetic vascular grafts: wrong models, wrong questions and no healing. Biomaterials. 2007;28:5009-27.

4. Hu WJ, Eaton JW, Tang LP. Molecular basis of biomaterial-mediated foreign body reactions. Blood. 2001;98:1231-8.

5. Wang X, Lin P, Yao Q, Chen C. Development of small-diameter vascular grafts. World J Surg. 2007;31:682-9.

6. Matsumura G, Hibino N, Ikada Y, Kurosawa H, Shin'oka T. Successful application of tissue engineered vascular autografts: clinical experience. Biomaterials. $2003 ; 24: 2303-8$

7. Hoerstrup SP, Cummings I, Lachat M, Schoen FJ, Jenni R, Leschka S, et al. Functional growth in tissue-engineered living, vascular grafts: follow-up at 100 weeks in a large animal model. Circulation. 2006;114:I159-66.

8. Meinhart JG, Deutsch M, Fischlein T, Howanietz N, Froschl A, Zilla P. Clinical autologous in vitro endothelialization of 153 infrainguinal ePTFE grafts. Ann Thorac Surg. 2001;71:S327-31.
9. L'Heureux N, Dusserre N, Konig G, Victor B, Keire P, Wight TN, et al. Human tissue-engineered blood vessels for adult arterial revascularization. Nat Med. 2006; 12:361-5.

10. Nottelet B, Pektok E, Mandracchia D, Tille JC, Walpoth B, Gurny R, et al. Factorial design optimization and in vivo feasibility of poly(epsilon-caprolactone)micro- and nanofiber-based small diameter vascular grafts. J Biomed Mater Res A. 2008;89:865-75.

11. Pektok E, Nottelet B, Tille JC, Gurny R, Kalangos A, Moeller M, et al. Degradation and healing characteristics of small-diameter poly(epsilon-caprolactone) vascular grafts in the rat systemic arterial circulation. Circulation. 2008;118: 2563-70.

12. de Valence S, Tille JC, Mugnai D, Mrowczynski W, Gurny R, Möller M, et al. Long term performance of polycaprolactone vascular grafts in a rat abdominal aorta replacement model. Biomaterials. 2012;33:38-47.

13. National Research Council. Guide for care and use of laboratory animals. Washington, DC: National Academy Press; 1996.

14. Association for the Advancement of Medical Instrumentation, American National Standards Institute. Cardiovascular implants: tubular vascular prostheses. Arlington, VA: Association for the Advancement of Medical Instrumentation; 2004.

15. Feldkamp LA, Davis LC, Kress JW. Practical cone-beam algorithm. J Opt Soc Am A. 1984;1:612-9.

16. Becker RC. Seminars in thrombosis, thrombolysis and vascular biology: 1 . The vascular endothelium. Cardiology. 1991;78:13-22.

17. Schoen FJ, Harasaki H, Kim KM, Anderson HC, Levy RJ. Biomaterial-associated calcification: pathology, mechanisms, and strategies for prevention. J Biomed Mater Res Appl Biomater. 1988;22:11-36.

18. Schoen FJ. Biomaterial-associated infection, neoplasia, and calcification. Clinicopathologic features and pathophysiologic concepts. ASAIO Trans. 1987;33: 8-18.

19. Tomizawa Y, Takanashi Y, Noishiki Y, Nishida H, Endo M, Koyanagi H. Evaluation of small caliber vascular prostheses implanted in small children: activated angiogenesis and accelerated calcification. ASAIO J. 1998;44:M496-500.

20. Salacinski HJ, Goldner S, Giudiceandrea A, Hamilton G, Seifalian AM, Edwards A, et al. The mechanical behavior of vascular grafts: a review. J Biomater Appl. 2001;15:241-78. 


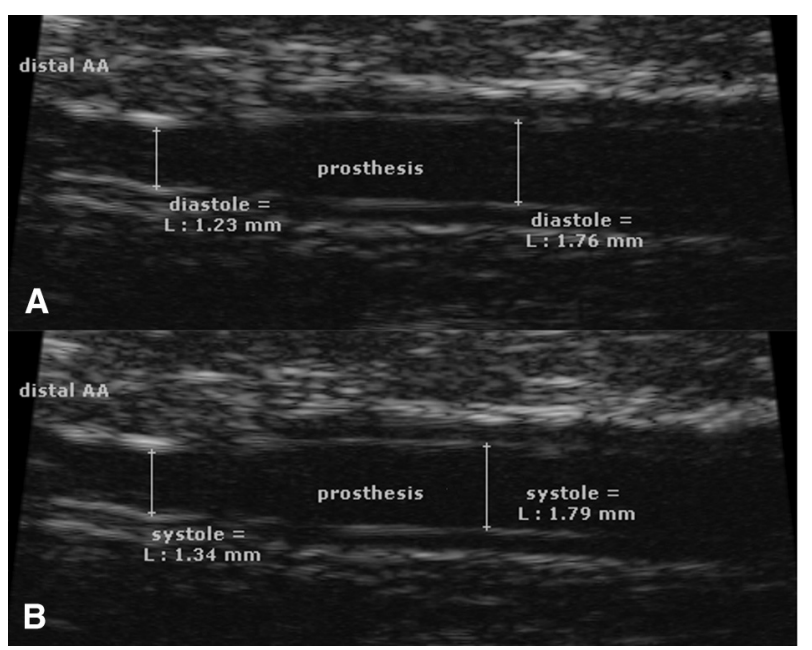

FIGURE E1. Evaluation of graft compliance. The inner diameter of polycaprolactone grafts and the adjacent abdominal aorta of a rat were measured in vivo from high-resolution ultrasound images during diastolic (A) and systolic (B) phases. Concomitant noninvasive systolic and diastolic blood pressures were recorded. $A A$, Aorta; $L$, length.

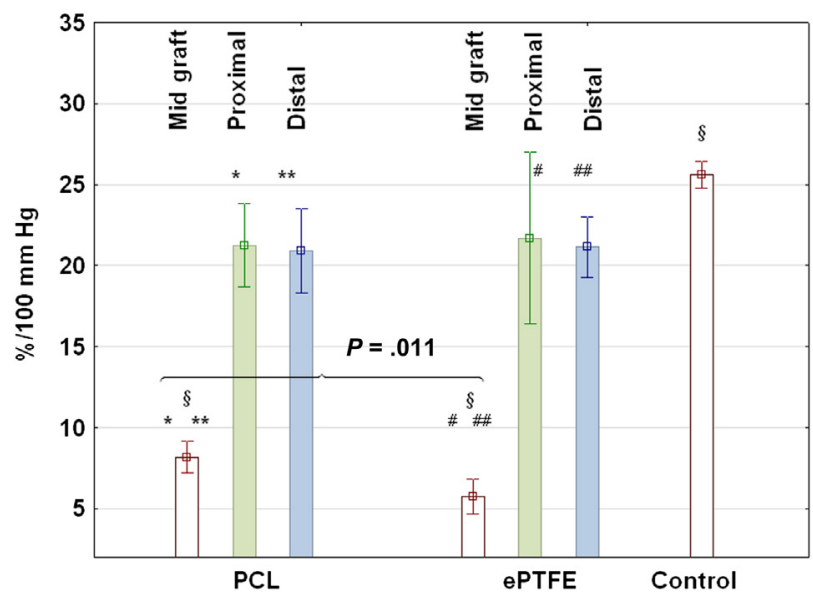

FIGURE E2. Results of in vivo compliance. The compliance of PCL implants was significantly greater than ePTFE grafts (mid graft). The compliance of both the PCL and PPTFE grafts were significantly less than adjacent parts of native aortas (proximal and distal) and control (nonoperated infrarenal abdominal aortas). $P C L$, Polycaprolactone; $e P T F E$, expanded polytetrafluoroethylene.*,**,\#,\#\#, P $^{*}<.01$.
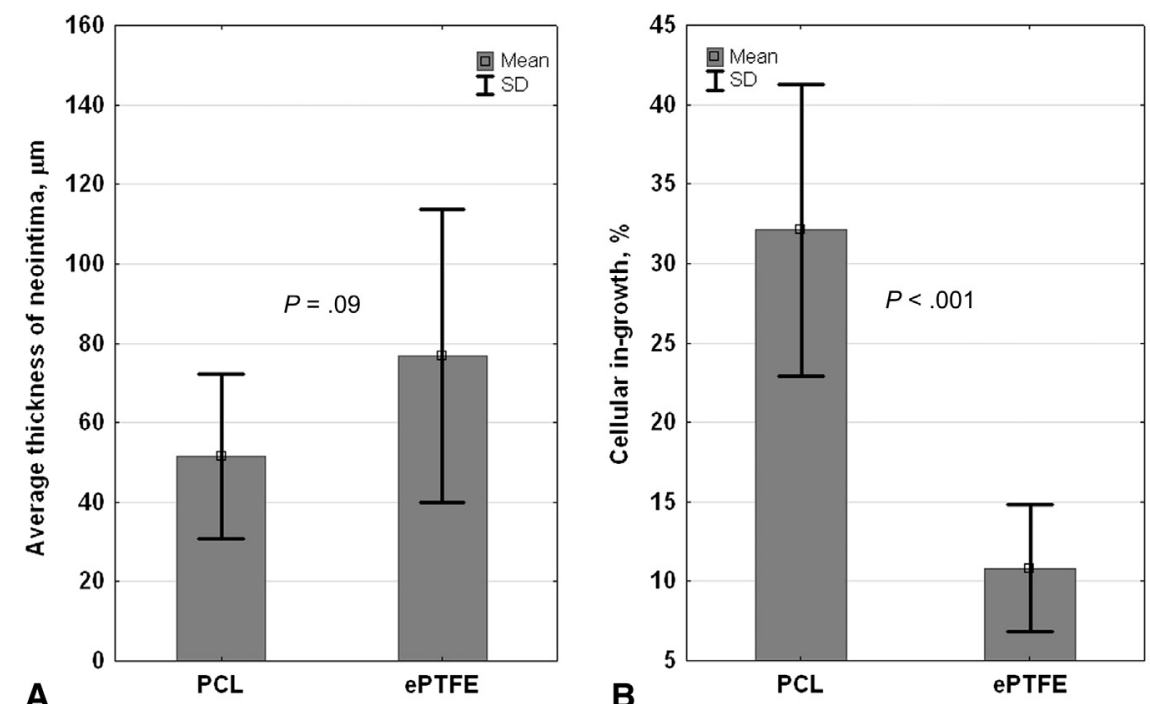

FIGURE E3. Results of morphometric analysis. (A) The average neointima thickness of PCL implants was significantly less than in ePTFE grafts. (B) The percent transmural cellular in-growth was greater in PCL than in ePTFE. PCL, Polycaprolactone; $e P T F E$, expanded polytetrafluoroethylene; SD, standard deviation. 